\title{
A class of integral operators from Lebesgue spaces into harmonic Bergman-Besov or weighted Bloch spaces
}

\author{
Ömer Faruk Doğan (1D

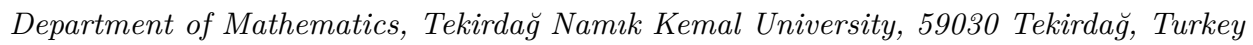

\begin{abstract}
We consider a class of two-parameter weighted integral operators induced by harmonic Bergman-Besov kernels on the unit ball of $\mathbb{R}^{n}$ and characterize precisely those that are bounded from Lebesgue spaces $L_{\alpha}^{p}$ into harmonic Bergman-Besov spaces $b_{\beta}^{q}$, weighted Bloch spaces $b_{\beta}^{\infty}$ or the space of bounded harmonic functions $h^{\infty}$, allowing the exponents to be different. These operators can be viewed as generalizations of the harmonic BergmanBesov projections.
\end{abstract}

Mathematics Subject Classification (2020). 47B34, 47G10, 31B05, 31B10, 42B35, $45 \mathrm{P} 05$

Keywords. integral operator, harmonic Bergman-Besov kernel, harmonic Bergman-Besov space, weighted harmonic Bloch space, harmonic Bergman-Besov projection

\section{Introduction and main results}

Let $n \geq 2$ be an integer and $\mathbb{B}=\mathbb{B}_{n}$ be the open unit ball in $\mathbb{R}^{n}$. Let $\nu$ be the Lebesgue volume measure on $\mathbb{B}$ normalized so that $\nu(\mathbb{B})=1$. For $\alpha \in \mathbb{R}$, we define the weighted volume measures $\nu_{\alpha}$ on $\mathbb{B}$ by

$$
d \nu_{\alpha}(x)=\frac{1}{V_{\alpha}}\left(1-|x|^{2}\right)^{\alpha} d \nu(x) .
$$

These measures are finite when $\alpha>-1$ and in this case we choose $V_{\alpha}$ so that $\nu_{\alpha}(\mathbb{B})=1$. Naturally $V_{0}=1$. For $\alpha \leq-1$, we set $V_{\alpha}=1$. We denote the Lebesgue classes with respect to $\nu_{\alpha}$ by $L_{\alpha}^{p}, 0<p<\infty$ and the corresponding norms by $\|\cdot\|_{L_{\alpha}^{p}}$.

Let $h(\mathbb{B})$ be the space of all complex-valued harmonic functions on $\mathbb{B}$ with the topology of uniform convergence on compact subsets. The space of bounded harmonic functions on $\mathbb{B}$ is denoted by $h^{\infty}$. For $0<p<\infty$ and $\alpha>-1$, the weighted harmonic Bergman space $b_{\alpha}^{p}$ is defined by $b_{\alpha}^{p}=L_{\alpha}^{p} \cap h(\mathbb{B})$ endowed with the norm $\|\cdot\|_{L_{\alpha}^{p}}$. The subfamily $b_{\alpha}^{2}$ is a reproducing kernel Hilbert space with respect to the inner product $[f, g]_{b_{\alpha}^{2}}=\int_{\mathbb{B}} f \bar{g} d \nu_{\alpha}(x)$ and with the reproducing kernel $R_{\alpha}(x, y)$ such that $f(x)=\left[f, R_{\alpha}(x, \cdot)\right]_{b_{\alpha}^{2}}$ for every $f \in b_{\alpha}^{2}$ and $x \in \mathbb{B}$. It is well-known that $R_{\alpha}$ is real-valued and $R_{\alpha}(x, y)=R_{\alpha}(y, x)$. The homogeneous

Email address: ofdogan@nku.edu.tr

Received: 13.07.2020; Accepted: 30.12.2020 
expansion of $R_{\alpha}(x, y)$ is given in the $\alpha>-1$ part of the formulas (1.2) and (1.3) below (see [2], [7]).

For $\alpha>-1$, the orthogonal projection $Q_{\alpha}: L_{\alpha}^{2} \rightarrow b_{\alpha}^{2}$ is given by the integral operator

$$
Q_{\alpha} f(x)=\frac{1}{V_{\alpha}} \int_{\mathbb{B}} R_{\alpha}(x, y) f(y)\left(1-|y|^{2}\right)^{\alpha} d \nu(y) \quad\left(f \in L_{\alpha}^{2}\right) .
$$

This integral operator plays a major role in the theory of weighted harmonic Bergman spaces and the question when Bergman projection $Q_{\alpha}: L_{\beta}^{p} \rightarrow b_{\beta}^{p}$ is bounded is studied in many sources such as ([8, Theorem 3.1], [11, Theorem 2.5], [12, Theorem 3.1]). By allowing the exponents, the weights and the parameters in the integrand to be different, our goal is to determine exactly when the integral operator in (1.1) is bounded from $L_{\alpha}^{p}$ to $b_{\beta}^{q}$.

Furthermore, we also remove the restriction $\alpha>-1$. The weighted harmonic Bergman spaces $b_{\alpha}^{p}$, initially defined for $\alpha>-1$, can be extended to the whole range $\alpha \in \mathbb{R}$. These are studied in detail in [7] and will be reviewed in Section 2. We call the extended family $b_{\alpha}^{p}(\alpha \in \mathbb{R})$ as harmonic Bergman-Besov spaces and the corresponding reproducing kernels as $R_{\alpha}(x, y)(\alpha \in \mathbb{R})$ harmonic Bergman-Besov kernels. The homogeneous expansion of $R_{\alpha}(x, y)$ can be expressed in terms of zonal harmonics

$$
R_{\alpha}(x, y)=\sum_{k=0}^{\infty} \gamma_{k}(\alpha) Z_{k}(x, y) \quad(\alpha \in \mathbb{R}, x, y \in \mathbb{B}),
$$

where (see [6, Theorem 3.7], [7, Theorem 1.3])

$$
\gamma_{k}(\alpha):= \begin{cases}\frac{(1+n / 2+\alpha)_{k}}{(n / 2)_{k}}, & \text { if } \alpha>-(1+n / 2) ; \\ \frac{(k !)^{2}}{(1-(n / 2+\alpha))_{k}(n / 2)_{k}}, & \text { if } \alpha \leq-(1+n / 2),\end{cases}
$$

and $(a)_{b}$ is the Pochhammer symbol. For definition and details about $Z_{k}(x, y)$, see [1, Chapter 5].

Finally, we allow the exponents $p, q$ to be $\infty$. We denote by $L^{\infty}=L^{\infty}(\nu)$ the Lebesgue class of all essentially bounded functions on $\mathbb{B}$ with respect to $\nu$. In this case we have $L^{\infty}\left(d \nu_{\alpha}\right)=L^{\infty}$ for every $\alpha \in \mathbb{R}$ and because of this we need to use a different weighted class. For $\alpha \in \mathbb{R}$, we define

$$
\mathcal{L}_{\alpha}^{\infty}:=\left\{\varphi \text { is measurable on } \mathbb{B}:\left(1-|x|^{2}\right)^{\alpha} \varphi(x) \in L^{\infty}\right\},
$$

so that $\mathcal{L}_{0}^{\infty}=L^{\infty}$. The norm on $\mathcal{L}_{\alpha}^{\infty}$ is

$$
\|\varphi\|_{\mathcal{L}_{\alpha}^{\infty}}=\left\|\left(1-|x|^{2}\right)^{\alpha} \varphi(x)\right\|_{L^{\infty}}
$$

For $\alpha>0$, the weighted harmonic Bloch space $b_{\alpha}$ is defined by $h(\mathbb{B}) \cap \mathcal{L}_{\alpha}^{\infty}$ and also can be extended to the whole range $\alpha \in \mathbb{R}$. The properties of this extended family are studied in detail in [5] and will be reviewed in Section 2.

We can now state our main result. For $b, c \in \mathbb{R}$ define the integral operator $T_{b c}$ by

$$
T_{b c} f(x)=\int_{\mathbb{B}} R_{c}(x, y) f(y)\left(1-|y|^{2}\right)^{b} d \nu(y) .
$$

Our aim is to determine exactly when $T_{b c}$ is bounded from $L_{\alpha}^{p}$ to $b_{\beta}^{q}$. The result is divided into two cases depending on whether $1 \leq q<\infty$ or $q=\infty$ that describe its boundedness in terms of the six parameters $(b, c, \alpha, \beta, p, q)$ involved.

Theorem 1.1. Let $b, c, \alpha, \beta \in \mathbb{R}, 1 \leq p \leq \infty$ and $1 \leq q<\infty$. Then $T_{b c}$ is bounded from $L_{\alpha}^{p}$ to $b_{\beta}^{q}\left(\mathcal{L}_{\alpha}^{\infty}\right.$ to $b_{\beta}^{q}$ when $\left.p=\infty\right)$ if and only if $(b, c, \alpha, \beta, p, q)$ satisfy one of the following conditions:

(i) If $1<p \leq q<\infty$, then $\alpha+1<p(b+1)$ and $c \leq b+\frac{n+\beta}{q}-\frac{n+\alpha}{p}$; 
(ii) If $1=p \leq q<\infty$, then $\alpha<b$ and $c \leq b+\frac{n+\beta}{q}-(n+\alpha)$ or $\alpha \leq b$ and $c<b+\frac{n+\beta}{q}-(n+\alpha)$

(iii) If $1 \leq q<p<\infty$, then $\alpha+1<p(b+1)$ and $c<b+\frac{1+\beta}{q}-\frac{1+\alpha}{p}$;

(iv) If $1 \leq q<p=\infty$, then $\alpha-1<b$ and $c<b+\frac{\beta+1}{q}-\alpha$.

Theorem 1.2. Let $b, c, \alpha, \beta \in \mathbb{R}$ and $1 \leq p \leq \infty$. Then $T_{b c}$ is bounded from $L_{\alpha}^{p}$ to $b_{\beta}^{\infty}$ $\left(\mathcal{L}_{\alpha}^{\infty}\right.$ to $b_{\beta}^{\infty}$ when $\left.p=\infty\right)$ if and only if $(b, c, \alpha, \beta, p)$ satisfy one of the following conditions:

(i) If $1<p<\infty$, then $\alpha+1<p(b+1)$ and $c \leq b+\beta-\frac{n+\alpha}{p}$;

(ii) If $p=1$, then $\alpha<b$ and $c \leq b+\beta-(n+\alpha)$ or $\alpha \leq b$ and $c<b+\beta-(n+\alpha)$;

(iii) If $p=\infty$, then $\alpha-1<b$ and $c \leq b+\beta-\alpha$.

Moreover, we also determine when $T_{b c}$ is bounded from $L_{\alpha}^{p}$ to $h^{\infty}$.

Theorem 1.3. Let $b, c, \alpha \in \mathbb{R}$ and $1 \leq p \leq \infty$. Then $T_{b c}$ is bounded from $L_{\alpha}^{p}$ to $h^{\infty}\left(\mathcal{L}_{\alpha}^{\infty}\right.$ to $h^{\infty}$ when $\left.p=\infty\right)$ if and only if $(b, c, \alpha, p)$ satisfy one of the following conditions:

(i) If $1<p<\infty$, then $\alpha+1<p(b+1)$ and $c<b-\frac{n+\alpha}{p}$;

(ii) If $p=1$, then $\alpha<b$ and $c \leq b-(n+\alpha)$ or $\alpha \leq b$ and $c<b-(n+\alpha)$;

(iii) If $p=\infty$, Then $\alpha-1<b$ and $c<b-\alpha$.

Harmonic Bergman-Besov projections on harmonic spaces have been studied for some time. See [7, Theorem 1.4] for $1 \leq p=q<\infty, b=c, \alpha=\beta \in \mathbb{R}$ and [5, Theorem 1.6] for $p=q=\infty, b=c, \alpha=\beta \in \mathbb{R}$. The other result we know of on Besov spaces is [9, Theorem 4.1] in which $1 \leq p=q \leq \infty, b=c>-1$ and $\alpha=\beta=-n$. Note that in our results we have $b, c, \alpha, \beta \in \mathbb{R}$ unrestrictedly and thus our operators in some sense generalize the harmonic Bergman-Besov projections. The holomorphic counterparts of our results on the boundedness of integral operators induced by holomorphic Bergman-Besov kernels appear in [10, Theorem 1.8 and Theorem 1.9].

For experts in analysis, one of the interesting problem might be the boundedness of $T_{b c}$ between different weighted Lebesgue classes. This problem is considered earlier in [4] as seven theorems that describe boundedness of $T_{b c}$ in terms of the six parameters $(b, c, \alpha, \beta, p, q)$ involved and the proof of our main results most heavily depends on these results. We combine all the seven theorems out there as two theorems below depending on the value of $q$. The following theorem is a combination of [4, Theorems 1.1, 1.2, 1,3 and $1.4]$ with $1 \leq q<\infty$. Note that they include an extra operator that replace $R_{c}(x, y)$ in the integral (1.4) by $\left|R_{c}(x, y)\right|$ because they need operators with positive kernels to apply Schur tests.

Theorem 1.4. Let $b, c, \alpha, \beta \in \mathbb{R}$ with $\beta>-1,1 \leq p \leq \infty$ and $1 \leq q<\infty$. Then $T_{b c}$ is bounded from $L_{\alpha}^{p}$ to $L_{\beta}^{q}\left(\mathcal{L}_{\alpha}^{\infty}\right.$ to $L_{\beta}^{q}$ when $\left.p=\infty\right)$ if and only if $(b, c, \alpha, \beta, p, q)$ satisfy one of the following conditions:

(i) If $1<p \leq q<\infty$, then $\alpha+1<p(b+1)$ and $c \leq b+\frac{n+\beta}{q}-\frac{n+\alpha}{p}$;

(ii) If $1=p \leq q<\infty$, then $\alpha<b$ and $c \leq b+\frac{n+\beta}{q}-(n+\alpha)$ or $\alpha \leq b$ and $c<b+\frac{n+\beta}{q}-(n+\alpha) ;$

(iii) If $1 \leq q<p<\infty$, then $\alpha+1<p(b+1)$ and $c<b+\frac{1+\beta}{q}-\frac{1+\alpha}{p}$; 
(iv) If $1 \leq q<p=\infty$, then $\alpha-1<b$ and $c<b+\frac{\beta+1}{q}-\alpha$.

The following theorem is a combination of [4, Theorems 1.4, 1.5 and 1.7] with $q=\infty$.

Theorem 1.5. Let $b, c, \alpha, \beta \in \mathbb{R}$ with $\beta \geq 0$ and $1 \leq p \leq \infty$. Then $T_{b c}$ is bounded from $L_{\alpha}^{p}$ to $\mathcal{L}_{\beta}^{\infty}\left(\mathcal{L}_{\alpha}^{\infty}\right.$ to $\mathcal{L}_{\beta}^{\infty}$ when $\left.p=\infty\right)$ if and only if $(b, c, \alpha, \beta, p)$ satisfy one of the following conditions:

(i) If $1<p<\infty$, then $\alpha+1<p(b+1)$ and $c \leq b+\beta-\frac{n+\alpha}{p}$, and the strict inequality holds when $\beta=0$;

(ii) If $p=1$, then $\alpha<b$ and $c \leq b+\beta-(n+\alpha)$ or $\alpha \leq b$ and $c<b+\beta-(n+\alpha)$;

(iii) If $p=\infty$, then $\alpha-1<b$ and $c \leq b+\beta-\alpha$, and the strict inequality holds when $\beta=0$.

The conditions $\beta>-1$ when $q<\infty$ in Theorem 1.4 and $\beta \geq 0$ when $q=\infty$ in Theorem 1.5 cannot be removed as explained in [4, Corollary 1.4]. Since we use repeatedly in this paper, this result is given again as Corollary 3.5 below. Notice that, our results are a variation of these theorems that removes the annoying conditions $\beta>-1$ when $q<\infty$ and $\beta \geq 0$ when $q=\infty$.

In Section 2, we collect some known facts about the harmonic Bergman-Besov and weighted Bloch spaces. Sections 3 and 4 are devoted to the proofs of Theorem 1.1 and Theorems 1.2 and 1.3 , respectively.

\section{Preliminaries}

For two positive expressions $X$ and $Y$, we write $X \lesssim Y$ if there exists a positive constant $C$, whose exact value is inessential, such that $X \leq C Y$. We also write $X \sim Y$ if both $X \lesssim Y$ and $Y \lesssim X$.

The Pochhammer symbol $(a)_{b}$ is defined by

$$
(a)_{b}=\frac{\Gamma(a+b)}{\Gamma(a)},
$$

when $a$ and $a+b$ are off the pole set $-\mathbb{N}$ of the gamma function. Stirling formula gives

$$
\frac{(a)_{c}}{(b)_{c}} \sim c^{a-b}, \quad c \rightarrow \infty .
$$

Let $\mathbb{S}$ be the unit sphere in $\mathbb{R}^{n}$ and $\sigma$ be the surface measure on $\mathbb{S}$ normalized so that $\sigma(\mathbb{S})=1$. For $f \in L_{0}^{1}$, the polar coordinates formula is

$$
\int_{\mathbb{B}} f(x) d \nu(x)=n \int_{0}^{1} \epsilon^{n-1} \int_{\mathbb{S}} f(\epsilon \zeta) d \sigma(\zeta) d \epsilon,
$$

in which $x=\epsilon \zeta$ with $\epsilon>0$ and $\zeta \in \mathbb{S}$.

We let $1 \leq p, p^{\prime} \leq \infty$ be the conjugate exponent. That is, if $1<p<\infty$, then $\frac{1}{p}+\frac{1}{p^{\prime}}=1$; if $p=1$, then $p^{\prime}=\infty$ and if $p=\infty$, then $p^{\prime}=1$.

We show an integral inner product on a function space $X$ by $[\cdot, \cdot]_{X}$.

In multi-index notation, $m=\left(m_{1}, \ldots, m_{n}\right)$ is an n-tuple of non-negative integers $m_{1}, \ldots, m_{n}$ and

$$
\partial^{m} f=\frac{\partial^{|m|} f}{\partial x_{1}^{m_{1}} \cdots \partial x_{n}^{m_{n}}}
$$

is the usual partial derivative for smooth $f$, where $|m|=m_{1}+\cdots+m_{n}$.

The weighted harmonic Bergman spaces $b_{\alpha}^{p}(\alpha>-1)$ can be extended to all $\alpha \in \mathbb{R}$. For $\alpha \in \mathbb{R}$ and $0<p<\infty$, let $N$ be a non-negative integer such that $\alpha+p N>-1$. The harmonic Bergman-Besov space $b_{\alpha}^{p}$ consists of all $f \in h(\mathbb{B})$ such that

$$
\left(1-|x|^{2}\right)^{N} \partial^{m} f \in L_{\alpha}^{p},
$$


for every multi-index $m$ with $|m|=N$. The space $b_{\alpha}^{p}$ does not depend on the choice of $N$ as long as $\alpha+p N>-1$ is satisfied.

Likewise, given $\alpha \in \mathbb{R}$, pick a non-negative integer $N$ such that $\alpha+N>0$. The weighted harmonic Bloch space $b_{\alpha}^{\infty}$ consists of all $f \in h(\mathbb{B})$ such that

$$
\left(1-|x|^{2}\right)^{N} \partial^{m} f \in \mathcal{L}_{\alpha}^{\infty},
$$

for every multi-index $m$ with $|m|=N$. When $\alpha=0$, one can choose $N=1$ and

$$
b_{0}^{\infty}=\left\{f \in h(\mathbb{B}): \sup _{x \in \mathbb{B}}\left(1-|x|^{2}\right)|\nabla f(x)|<\infty\right\} .
$$

This is the most studied member of the family. As before, the spaces $b_{\alpha}^{\infty}$ do not depend on the choice of $N$ as long as $\alpha+N>0$ is satisfied

In the definitions of $b_{\alpha}^{p}$ and $b_{\alpha}^{\infty}$, instead of partial derivatives one can use more effectively certain radial differential operators $D_{s}^{t}: h(\mathbb{B}) \rightarrow h(\mathbb{B}),(s, t \in \mathbb{R})$ defined in terms of reproducing kernels of harmonic Bergman spaces that are introduced in [6] and [7].

Before going to the definition, note that for every $\alpha \in \mathbb{R}$ we have $\gamma_{0}(\alpha)=1$, and therefore

$$
R_{\alpha}(x, 0)=R_{\alpha}(0, y)=1, \quad(x, y \in \mathbb{B}, \alpha \in \mathbb{R}) .
$$

Checking the two cases in (1.3), we have by (2.1)

$$
\gamma_{k}(\alpha) \sim k^{1+\alpha} \quad(k \rightarrow \infty)
$$

$R_{\alpha}(x, y)$ is harmonic as a function of either of its variables on $\overline{\mathbb{B}}$.

For any $f \in h(\mathbb{B})$ there exist homogeneous harmonic polynomials $f_{k}$ of degree $k$ such that $f=\sum_{k=0}^{\infty} f_{k}$, the series converging absolutely and uniformly on compact subsets of $\mathbb{B}$ which is called the homogeneous expansion of $f$ (see [1]).

Definition 2.1. Let $f=\sum_{k=0}^{\infty} f_{k} \in h(\mathbb{B})$ be given by its homogeneous expansion. For $s, t \in \mathbb{R}$ we define $D_{s}^{t}$ on $h(\mathbb{B})$ by

$$
D_{s}^{t} f:=\sum_{k=0}^{\infty} \frac{\gamma_{k}(s+t)}{\gamma_{k}(s)} f_{k}
$$

By $(2.3), \gamma_{k}(s+t) / \gamma_{k}(s) \sim k^{t}$ for any $s, t$ and, roughly speaking, $D_{s}^{t}$ multiplies the $k$ th homogeneous part of $f$ by $k^{t}$. For every $s \in \mathbb{R}, D_{s}^{0}=I$, the identity. An important property of $D_{s}^{t}$ is that it is invertible with two-sided inverse $D_{s+t}^{-t}$ :

$$
D_{s+t}^{-t} D_{s}^{t}=D_{s}^{t} D_{s+t}^{-t}=I
$$

which follows from the additive property $D_{s+t}^{z} D_{s}^{t}=D_{s}^{z+t}$.

For every $s, t \in \mathbb{R}$, the map $D_{s}^{t}: h(\mathbb{B}) \rightarrow h(\mathbb{B})$ is continuous in the topology of uniform convergence on compact subsets (see [7, Theorem 3.2]). The parameter $s$ plays a minor role and is used to have the precise relation $D_{s}^{t} R_{s}(x, y)=R_{s+t}(x, y)$.

Consider the linear transformation $I_{s}^{t}$ defined for $f \in h(\mathbb{B})$ by

$$
I_{s}^{t} f(x):=\left(1-|x|^{2}\right)^{t} D_{s}^{t} f(x) .
$$

The spaces $b_{\alpha}^{p}$ and $b_{\alpha}^{\infty}$ can equivalently be defined by using the operators $D_{s}^{t}$.

Definition 2.2. For $0<p<\infty$ and $\alpha \in \mathbb{R}$, we define the harmonic Bergman-Besov space $b_{\alpha}^{p}$ to consist of all $f \in h(\mathbb{B})$ for which $I_{s}^{t} f$ belongs to $L_{\alpha}^{p}$ for some $s, t$ satisfying (see [7] when $1 \leq p<\infty$, and [3] when $0<p<1$ )

$$
\alpha+p t>-1 \text {. }
$$

The quantity

$$
\|f\|_{b_{\alpha}^{p}}^{p}=\left\|I_{s}^{t} f\right\|_{L_{\alpha}^{p}}^{p}=c_{\alpha} \int_{\mathbb{B}}\left|D_{s}^{t} f(x)\right|^{p}\left(1-|x|^{2}\right)^{\alpha+p t} d \nu(x)<\infty
$$

defines a norm (quasinorm when $0<p<1$ ) on $b_{\alpha}^{p}$ for any such $s, t$. 
Definition 2.3. For $\alpha \in \mathbb{R}$, we define the harmonic Bloch space $b_{\alpha}^{\infty}$ to consist of all $f \in h(\mathbb{B})$ for which $I_{s}^{t} f$ belongs to $\mathcal{L}_{\alpha}^{\infty}$ for some $s, t$ satisfying (see [5])

$$
\alpha+t>0 \text {. }
$$

The quantity

$$
\|f\|_{b_{\alpha}^{\infty}}=\left\|I_{s}^{t} f\right\|_{L_{\alpha}^{\infty}}^{p}=\sup _{x \in \mathbb{B}}\left(1-|x|^{2}\right)^{\alpha+t}\left|D_{s}^{t} f(x)\right|<\infty
$$

defines a norm on $b_{\alpha}^{\infty}$ for any such $s, t$.

It is well-known that Definitions 2.2 and 2.3 are independent of $s, t$ under (2.4) and (2.5), respectively and the norms (quasinorms when $0<p<1$ ) on a given space are all equivalent. Thus for a given pair $s, t, I_{s}^{t}$ isometrically imbeds $b_{\alpha}^{p}$ into $L_{\alpha}^{p}$ if and only if (2.4) holds, and $I_{s}^{t}$ isometrically imbeds $b_{\alpha}^{\infty}$ into $L_{\alpha}^{\infty}$ if and only if (2.5) holds.

The most significant property of the operators $D_{s}^{t}$ is that it allows us to pass from one Bergman-Besov (or weighted Bloch) space to another. Actually, we have the following isomorphisms.

Lemma 2.4. Let $0<p<\infty$ and $\alpha, s, t \in \mathbb{R}$.

(i) The map $D_{s}^{t}: b_{\alpha}^{p} \rightarrow b_{\alpha+p t}^{p}$ is an isomorphism.

(ii) The map $D_{s}^{t}: b_{\alpha}^{\infty} \rightarrow b_{\alpha+t}^{\infty}$ is an isomorphism.

For a proof of part (i) of the above lemma see [7, Corollary 9.2] when $1 \leq p<\infty$ and [3, Proposition 4.7] when $0<p<1$. For part (ii) see [5, Proposition 4.6].

The lemma below shows that if $x$ stays close to 0 , then $R_{\alpha}(x, y)$ is uniformly away from 0 for every $y \in \mathbb{B}$.

Lemma 2.5 ([5, Lemma 3.2]). Let $\alpha \in \mathbb{R}$. There exists $\epsilon>0$ such that for all $|x|<\epsilon$ and for all $y \in \mathbb{B}$, we have $R_{\alpha}(x, y) \geq 1 / 2$.

\section{Proof of Theorem 1.1}

In this section, we prove Theorem 1.1. Note that we call the second and third inequality in each of the four parts of Theorem 1.1 the first and second necessary condition, respectively.

Before the proof, we formulate the behavior of the operators $T_{b c}$ in many important situations which will be used in this and next section. These are from Section 3 of [4] and adapted from similar results in Section 4 of [10]. We begin by inserting some obvious inequalities that we use many times. If $a_{1}<a_{2}, u>0$, and $v \in \mathbb{R}$, then for $0 \leq t<1$,

$$
\left(1-t^{2}\right)^{a_{2}} \leq\left(1-t^{2}\right)^{a_{1}} \quad \text { and } \quad\left(1-t^{2}\right)^{u}\left(1+\log \left(1-t^{2}\right)^{-1}\right)^{-v} \lesssim 1 .
$$

The second inequality above leads to the following estimate.

Lemma 3.1 ([4, Lemma 3.1]). For $u, v \in \mathbb{R}$,

$$
\int_{0}^{1}\left(1-t^{2}\right)^{u}\left(1+\log \frac{1}{1-t^{2}}\right)^{-v} d t<\infty
$$

if $u>-1$ or $u=-1$ and $v>1$, and the integral diverges otherwise.

We will use the functions

$$
f_{u v}(x)=\left(1-|x|^{2}\right)^{u}\left(1+\log \frac{1}{1-|x|^{2}}\right)^{-v} \quad(u, v \in \mathbb{R})
$$

as test functions to obtain some of the necessary conditions of our theorems from the action of $T_{b c}$ on them.

Lemma 3.2 ([4, Lemma 3.2]). For $1 \leq p<\infty$, we have $f_{u v} \in L_{\alpha}^{p}$ if and only if $\alpha+p u>$ -1 , or $\alpha+p u=-1$ and $p v>1$. For $p=\infty$, we have $f_{u v} \in \mathcal{L}_{\alpha}^{\infty}$ if and only if $\alpha+u>0$, or $u=-\alpha$ and $v \geq 0$. 
Lemma 3.3 ([4, Lemma 3.3]). If $b+u>-1$ or if $b+u=-1$ and $v>1$, then $T_{b c} f_{u v}$ is a finite positive constant. Otherwise, $T_{b c} f_{u v}(x)=\infty$ for $|x| \leq \epsilon$, where $\epsilon$ is as in Lemma 2.5.

Proof of Theorem 1.1. Let $b, c, \alpha, \beta \in \mathbb{R}, 1 \leq p \leq \infty$ and $1 \leq q<\infty$.

First Necessary Condition. Assume that $T_{b c}$ is bounded from $L_{\alpha}^{p}$ to $b_{\beta}^{q}$. The proof can be handled in three cases depending on the value of $p$.

We first show the case $1<p<\infty$. Consider $f_{u v}$ with $u=-(1+\alpha) / p$ and $v=1$ so that $f_{u v} \in L_{\alpha}^{p}$ by Lemma 3.2. Then it is clear that $T_{b c} f_{u v} \in b_{\beta}^{q}$ and this implies $T_{b c} f_{u v}(0) \in \mathbb{C}$. We have by $(2.2)$

$$
T_{b c} f_{u v}(0)=\int_{\mathbb{B}}\left(1-|x|^{2}\right)^{b-(1+\alpha) / p}\left(1+\log \frac{1}{(1-|x|)^{2}}\right)^{-1} d \nu(x) .
$$

Writing the integral in polar coordinates and using Lemma 3.1, we obtain $(1+\alpha) / p<1+b$. Thus we derive the second inequality in parts (i) and (iii).

Next, we show the second case $p=1$. Consider $f_{u v}$ with $u>-(1+\alpha)$ and $v=0$ so that $f_{u 0} \in L_{\alpha}^{1}$ by Lemma 3.2. Then $T_{b c} f_{u 0} \in b_{\beta}^{q}$ and this implies $T_{b c} f_{u 0}(0) \in \mathbb{C}$. We have by $(2.2)$

$$
T_{b c} f_{u 0}(0)=\int_{\mathbb{B}}\left(1-|x|^{2}\right)^{b+u} d \nu(x)
$$

with $u>-(1+\alpha)$. Again writing the integral in polar coordinates and using Lemma 3.1, we obtain $\alpha \leq b$. Thus we derive the second inequality in part (ii).

The last case is $p=\infty$. Choose $u=-\alpha$ and $v=0$ so that $f_{u 0} \in \mathcal{L}_{\alpha}^{\infty}$ by Lemma 3.2. Then $T_{b c} f_{u 0} \in b_{\beta}^{q}$ and this implies $T_{b c} f_{u 0}(0) \in \mathbb{C}$. We have by (2.2) again,

$$
T_{b c} f_{u 0}(0)=\int_{\mathbb{B}}\left(1-|x|^{2}\right)^{b-\alpha} d \nu(x) .
$$

One more time writing the integral in polar coordinates and using Lemma 3.1, we obtain $b-\alpha>-1$. Finally, we derive the second inequality in part (iv).

Now we will show the Second Necessary Condition. We do this by composing bounded maps. Firstly, we present the following lemma which is a crucial component of this technique and will allow us to push $D_{s}^{t}$ into the integral operator $T_{b c}$.

Lemma 3.4. Let $c, b \in \mathbb{R}$ and $f \in L_{b}^{1}$. Then $D_{c}^{t} T_{b c} f=T_{b, c+t} f$ for every $t \in \mathbb{R}$.

Proof. Writing $T_{b c} f$ explicitly, it is easy to see that the proof can be verified in the same way as in [5, Lemma 2.3]. Thus we omit the details.

We also need the following result of [4].

Corollary 3.5 ([4, Corollary 3.6]). If $T_{b c}: L_{\alpha}^{p} \rightarrow L_{\beta}^{q}$ is bounded and $f \in L_{\alpha}^{p}$, then $g=T_{b c} f$ is harmonic on $\mathbb{B}$. If also $q<\infty$, then $\beta>-1$. Therefore $T_{b c}: L_{\alpha}^{p} \rightarrow b_{\beta}^{q}$ when it is bounded with $\beta>-1$ and $q<\infty$. Moreover, if $\beta \leq-1$ and $q<\infty$, then $T_{b c}: L_{\alpha}^{p} \rightarrow L_{\beta}^{q}$ is not bounded. On the other hand, if $T_{b c}: L_{\alpha}^{p} \rightarrow L^{\infty}$ is bounded and $f \in L_{\alpha}^{p}$, then $g=T_{b c} f \in h^{\infty}$. Finally, if $T_{b c}: L_{\alpha}^{p} \rightarrow \mathcal{L}_{\beta}^{\infty}$ is bounded, $f \in L_{\alpha}^{p}$, and $\beta>0$ then $g=T_{b c} f \in b_{\beta}^{\infty}$. Moreover, if $\beta<0$, then $T_{b c}: L_{\alpha}^{p} \rightarrow \mathcal{L}_{\beta}^{\infty}$ is not bounded.

Second Necessary Condition. We assume that $T_{b c}$ is bounded and the first necessary condition holds, and then we apply Theorem 1.4. Let $f \in L_{\alpha}^{p}$. We first show also $f \in L_{b}^{1}$ in order to able to use Lemma 3.4. In the first case $1<p<\infty$, by the Hölder inequality, we have

$$
\begin{aligned}
\|f\|_{L_{b}^{1}} & =\frac{V_{\alpha}}{V_{b}} \int_{\mathbb{B}}|f(x)|\left(1-|x|^{2}\right)^{b-\alpha} d \nu_{\alpha}(x) \\
& \lesssim\|f\|_{L_{\alpha}^{p}}\left(\int_{\mathbb{B}}\left(1-|x|^{2}\right)^{(b-\alpha) p^{\prime}} d \nu_{\alpha}(x)\right)^{1 / p^{\prime}}<\infty
\end{aligned}
$$


where the last inequality holds because $(b-\alpha) p^{\prime}+\alpha=\frac{p(1+b)-(\alpha+p)}{p-1}>\frac{1+\alpha-(\alpha+p)}{p-1}=-1$ by the already obtained first necessary condition. In the second case $p=1$, we have $\alpha \leq b$ by the first necessary condition and thus $f \in L_{b}^{1}$ by (3.1). In the third case $p=\infty, f \in \mathcal{L}_{\alpha}^{\infty}$ and the first necessary condition gives $-1<b-\alpha$; thus

$$
\begin{aligned}
\|f\|_{L_{b}^{1}} & =\frac{1}{V_{b}} \int_{\mathbb{B}}\left(1-|x|^{2}\right)^{\alpha}|f(x)|\left(1-|x|^{2}\right)^{b-\alpha} d \nu(x) \\
& \lesssim\|f\|_{\mathcal{L}_{\alpha}^{\infty}}\left(\int_{\mathbb{B}}\left(1-|x|^{2}\right)^{b-\alpha} d \nu(x)\right)<\infty
\end{aligned}
$$

and $f \in L_{b}^{1}$ again.

Now consider the composition of bounded maps

$$
L_{\alpha}^{p} \stackrel{\mathrm{T}_{\mathrm{bc}}}{\longrightarrow} b_{\beta}^{q} \stackrel{\mathrm{D}_{\mathrm{c}}^{-\beta / \mathrm{q}}}{\longrightarrow} b_{0}^{q},
$$

where Lemma 2.4 (i) is used. With $L_{\alpha}^{p} \subset L_{b}^{1}$ at hand from the above arguments, this composition equals $T_{b, c-\beta / q}$ by Lemma 3.4. We conclude that $T_{b, c-\beta / q}: L_{\alpha}^{p} \rightarrow L^{q}$ is bounded by Corollary 3.5. Hence we obtain the third inequalities in all parts of the theorem with $c-\beta / q$ in place of $c$ and 0 in place of $\beta$. But these are precisely the same third inequalities in all parts of the theorem.

Sufficiency. We assume that the three inequalities in all parts of the theorem hold. Notice that the third inequality is equivalent to that with $c$ replaced by $c-\beta / q$ and $\beta$ by 0 such as in the above paragraph. Now Theorem 1.4 and Corollary 3.5 imply that $T_{b, c-\beta / q}: L_{\alpha}^{p} \rightarrow L^{q} \cap h(\mathbb{B})=b^{q}$ is bounded. Then the composition of maps

$$
L_{\alpha}^{p} \stackrel{\mathrm{T}_{\mathrm{b}, \mathrm{c}-\beta / \mathrm{q}}}{\longrightarrow} b^{q} \stackrel{\mathrm{D}_{\mathrm{c}-\beta / \mathrm{q}}^{\beta / \mathrm{q}}}{\longrightarrow} b_{\beta}^{q}
$$

is also bounded by Lemma 2.4 (i). As in the proof of the second necessary condition above, we have $L_{\alpha}^{p} \subset L_{b}^{1}$. Thus by Lemma 3.4, this composition equals $T_{b c}: L_{\alpha}^{p} \rightarrow b_{\beta}^{q}$.

\section{Proofs of Theorems 1.2 and 1.3}

In this section, we prove Theorems 1.2 and 1.3. Once more we call the second and third inequality in each of the three parts of Theorems 1.2 and 1.3 the first and second necessary condition, respectively.

Proof of Theorem 1.2. Let $b, c, \alpha, \beta \in \mathbb{R}$ and $1 \leq p \leq q=\infty$. First Necessary Condition. Assume that $T_{b c}$ is bounded from $L_{\alpha}^{p}$ to $b_{\beta}^{\infty}$. We argue as in the proof of Theorem 1.1. We divide the proof in same three cases depending on the value of $p$ and use the same test functions $f_{u v}$ for each case obtaining $T_{b c} f_{u v} \in b_{\beta}^{\infty}$. Then $T_{b c} f_{u v}(0) \in \mathbb{C}$. Thus the second inequality in all parts of the theorem can be verified by repeating the first part of the above proof.

Second Necessary Condition. We assume that $T_{b c}$ is bounded and the first necessary condition holds, and then we use Theorem 1.5. Let $f \in L_{\alpha}^{p}$. With making small modifications in the second necessary part of above proof one can easily verify $f \in L_{b}^{1}$ again.

Now consider the composition of bounded maps

$$
L_{\alpha}^{p} \stackrel{\mathrm{T}_{b c}}{\longrightarrow} b_{\beta}^{\infty} \stackrel{\mathrm{D}_{\mathrm{c}}^{-\beta+1}}{\longrightarrow} b_{1}^{\infty},
$$

where this time Lemma 2.4 (ii) is used. With $L_{\alpha}^{p} \subset L_{b}^{1}$ at hand, the composition equals $T_{b, c-\beta+1}$ by Lemma 3.4. We conclude that $T_{b, c-\beta+1}: L_{\alpha}^{p} \rightarrow \mathcal{L}_{1}^{\infty}$ is bounded by Corollary 3.5. The third inequalities in Theorem 1.5 with $c-\beta+1$ in place of $c$ and 1 in place of $\beta$ give the third inequalities in Theorem 1.2.

Sufficiency. We assume that the three inequalities in all parts of the theorem hold. Notice that the third inequality is equivalent to that with $c$ replaced by $c-\beta+1$ and 
$\beta$ by 1 such as in the above paragraph. Now Theorem 1.5 and Corollary 3.5 imply that $T_{b, c-\beta+1}: L_{\alpha}^{p} \rightarrow \mathcal{L}_{1}^{\infty} \cap h(\mathbb{B})=b_{1}^{\infty}$ is bounded. Then the composition of maps

$$
L_{\alpha}^{p} \stackrel{\mathrm{T}_{\mathrm{b}, \mathrm{c}-\beta+1}}{\longrightarrow} b_{1}^{\infty} \stackrel{\mathrm{D}_{\mathrm{c}-\beta+1}^{\beta-1}}{\longrightarrow} b_{\beta}^{\infty}
$$

is also bounded by Lemma 2.4 (ii). The second inequality in all parts of the theorem such as in the proof of second necessity condition mentioned already yields $L_{\alpha}^{p} \subset L_{b}^{1}$. Thus by Lemma 3.4, this composition equals $T_{b c}: L_{\alpha}^{p} \rightarrow b_{\beta}^{\infty}$ is bounded.

Proof of Theorem 1.3. Assume that $T_{b c}: L_{\alpha}^{p} \rightarrow h^{\infty}$ is bounded. Then since $h^{\infty} \subset L^{\infty}$ with the same norms, $T_{b c}: L_{\alpha}^{p} \rightarrow L^{\infty}$ is also bounded. Therefore Theorem 1.5 implies the two necessary conditions in all three parts of the theorem hold.

Conversely, if both first and second necessary condition in all three parts of the theorem hold, then $T_{b c}: L_{\alpha}^{p} \rightarrow L^{\infty}$ is bounded by Theorem 1.5. But Corollary 3.5 shows that the range of $T_{b c}$ lies in $h^{\infty}$ rendering $T_{b c}: L_{\alpha}^{p} \rightarrow h^{\infty}$ is bounded.

Finally, a question runs through one's mind whether or not our results on weighted spaces can be obtained from those on unweighted spaces with $\alpha=\beta=0$. It turns out that they can and we now explain how. We assume that Theorems 1.1 and 1.2 are proved in the case $\alpha=\beta=0$ and we obtain them in the general case with nonzero $\alpha, \beta$. We go into details only for part (i) of Theorem 1.1. We need some results from the previous sections whose proofs are independent of the proofs of our main results. Let $M_{v}$ denotes the operator of multiplication by $\left(1-|x|^{2}\right)^{v}$. Obviously, $M_{-\alpha / p}: L^{p} \rightarrow L_{\alpha}^{p}$ is an isomorphism. Now, we will consider the sequence of the bounded maps

$$
L^{p} \stackrel{\mathrm{M}_{-\alpha / \mathrm{p}}}{\longrightarrow} L_{\alpha}^{p} \stackrel{\mathrm{T}_{\mathrm{bc}}}{\longrightarrow} b_{\beta}^{q} \stackrel{\mathrm{D}_{\mathrm{c}}^{-\beta / \mathrm{q}}}{\longrightarrow} b^{q},
$$

where the last map is also an isomorphism by Lemma 2.4 (i). The composition of these maps is $T_{b-\alpha / p, c-\beta / q}: L^{p} \rightarrow b^{q}$ by Lemma 3.4, and it is bounded if and only if the second map $T_{b c}: L_{\alpha}^{p} \rightarrow b_{\beta}^{q}$ is bounded. By assumption, the composition is bounded if and only if

$$
1<p(b+1)-\alpha \quad \text { and } \quad c-\frac{\beta}{q} \leq b-\frac{\alpha}{p}+n\left(\frac{1}{q}-\frac{1}{p}\right),
$$

which are nothing but the inequalities in part (i) of Theorem 1.1.

However, our proofs are not simplified significantly with $\alpha=\beta=0$. The classifications in Theorems 1.1-1.5 are according to $p, q$ and there seems to be no simple way of reducing them to fewer parts, because the inequalities in all seven parts can change between $<$ and $\leq$ without any apparent reason with $p, q$.

\section{References}

[1] S. Axler, P. Bourdon and W. Ramey, Harmonic function theory, 2nd ed., Grad. Texts in Math., 137, Springer, New York, 2001.

[2] A.E. Djrbashian and F.A. Shamoian, Topics in the Theory of $A_{\alpha}^{p}$ Spaces, Teubner Texts in Mathematics 105, Leipzig, 1988.

[3] Ö.F. Doğan, Harmonic Besov spaces with small exponents, Complex Var. Elliptic Equ. 65 (6), 1051-1075, 2020.

[4] Ö.F. Doğan, A Class of Integral Operators Induced by Harmonic Bergman-Besov Kernels on Lebesgue Classes, arXiv:2002.03193v2 [math.CA], 2020.

[5] Ö.F. Doğan and A.E. Üreyen, Weighted harmonic Bloch spaces on the ball, Complex Anal. Oper. Theory 12 (5), 1143-1177, 2018.

[6] S. Gergün, H.T. Kaptanoğlu and A.E. Üreyen, Reproducing kernels for harmonic Besov spaces on the ball, C. R. Math. Acad. Sci. Paris 347, 735-738, 2009. 
[7] S. Gergün, H.T. Kaptanoğlu and A.E. Üreyen, Harmonic Besov spaces on the ball, Int. J. Math. 27 (9), 1650070, 59 pp., 2016.

[8] M. Jevtić and M. Pavlović, Harmonic Bergman functions on the unit ball in $\mathbb{R}^{n}$, Acta Math. Hungar. 85, 81-96, 1999.

[9] M. Jevtić and M. Pavlović, Harmonic Besov spaces on the unit ball in $\mathbb{R}^{n}$, Rocky Mountain J. Math. 31, 1305-1316, 2001.

[10] H.T. Kaptanoğlu and A.E. Üreyen, Singular integral operators with Bergman-Besov kernels on the ball, Integr. Equ. Oper. Theory 91, 30 pp., 2019.

[11] S. Pérez-Esteva, Duality on vector-valued weighted harmonic Bergman spaces, Studia Math. 118, 37-47, 1996.

[12] K. Stroethoff, Harmonic Bergman spaces, in Holomorphic Spaces, Mathematical Sciences Research Institute Publications, 33, Cambridge University, Cambridge, 5163,1998 